Article

\title{
Terpene Glycosides from the Roots of Sanguisorba officinalis L. and Their Hemostatic Activities
}

\author{
Wei Sun, Zi-Long Zhang, Xin Liu, Shuang Zhang, Lu He, Zhe Wang and Guang-Shu Wang * \\ School of Pharmaceutical Sciences, Jilin University, Changchun 130021, China \\ * Author to whom correspondence should be addressed; E-Mail: wgs@jlu.edu.cn; \\ Tel.: +86-431-8561-9706.
}

Received: 15 May 2012; in revised form: 13 June 2012 / Accepted: 15 June 2012 /

Published: 25 June 2012

\begin{abstract}
Guided by a hemostasis bioassay, seven terpene glycosides were isolated from the roots of Sanguisorba officinalis L. by silica gel column chromatography and preparative HPLC. On the grounds of chemical and spectroscopic methods, their structures were identified as citronellol-1-O- $\alpha$-L-arabinofuranosyl-( $1 \rightarrow 6)-\beta$-D-glucopyranoside $(\mathbf{1})$, geraniol-1- $O$ - $\alpha$-L-arabinofuranosyl-( $1 \rightarrow 6)-\beta$-D-glucopyranoside $\quad(2), \quad$ geraniol-1- $O-\alpha$-Larabinopyranosyl-( $1 \rightarrow 6)-\beta$-D-glucopyranoside $\quad(3), \quad 3 \beta$-[( $\alpha$-L-arabinopyranosyl)oxy]-19 $\alpha$ hydroxyolean-12-en-28-oic acid 28- $\beta$-D-glucopyranoside (4), 3 $\beta$-[( $\alpha$-L-arabinopyranosyl)oxy]-19 $\alpha$-hydroxyurs-12-en-28-oic acid 28- $\beta$-D-glucopyranoside (ziyu-glycoside I, 5),

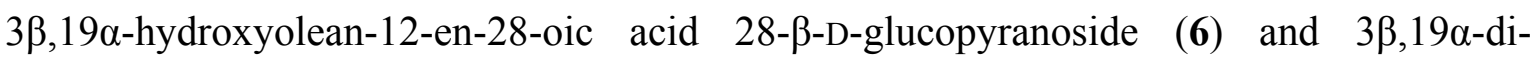
hydroxyurs-12-en-28-oic acid 28- $\beta$-D-glucopyranoside (7). Compound $\mathbf{1}$ is a new mono-terpene glycoside and compounds 2, 3 and $\mathbf{5}$ were isolated from the Sanguisorba genus for the first time. Compounds 1-7 were assayed for their hemostatic activities with a Goat Anti-Human a2-plasmin inhibitor ELISA kit, and ziyu-glycoside I (5) showed the strongest hemostatic activity among the seven terpene glycosides. This is the first report that ziyu-glycoside I has strong hemostatic activity.
\end{abstract}

Keywords: Sanguisorba officinalis; ziyu-glycoside I; citronellol; geraniol; hemostasis

\section{Introduction}

Sanguisorba officinalis L. (Rosaceae) is a perennial plant widely distributed in China, and its roots have been used as a traditional Chinese medicine for the treatment of hemostasis and inflammation [1]. 
A variety of chemical constituents, including tannins, triterpenoids, flavonoids, anthraquinones, steroids were isolated from S. officinalis L., and pharmacological studies on its hemostatic and antiinflammatory properties have been reported [2,3], but the molecular level mechanisms of these activities have not been reported until now. In order to study the mechanism of hemostasis, we have carried out the bioassay-guided isolation and identification of hemostatic constituents of the roots of S. officinalis L. In the present study, we report the isolation and identification of a new monoterpene glycoside 1, together with two known monoterpene glycosides 2, 3 and four known triterpenoids 4-7, and their hemostatic activities.

\section{Results and Discussion}

Compound 1, a colorless amorphous powder, produced a positive reaction to Molish reagent, and had the molecular formula $\mathrm{C}_{21} \mathrm{H}_{38} \mathrm{O}_{10}$ as determined by HRESIMS ([M+Na $]^{+} \mathrm{m} / z$ 473.2369). Its IR spectrum indicated the presence of hydroxyl $\left(3450 \mathrm{~cm}^{-1}\right)$ groups. Acid hydrolysis of compound 1 gave D-glucose and L-arabinose. The ${ }^{1} \mathrm{H}-,{ }^{13} \mathrm{C}$ - and DEPT-NMR spectrum (DMSO- $\mathrm{d}_{6}$ ) of compound $\mathbf{1}$ showed signals of a monoterpene moiety consisting of two singlets and one double methyl groups $\left[\delta_{\mathrm{H}} 1.64(3 \mathrm{H}, \mathrm{s}, \mathrm{H}-8), 1.56(3 \mathrm{H}, \mathrm{s}, \mathrm{H}-9)\right.$, and $0.85(3 \mathrm{H}, \mathrm{d}, J=6.4 \mathrm{~Hz} \mathrm{H}-10) ; \delta_{\mathrm{C}} 25.5$ (C-8), 17.5 (C-9), and 19.3 (C-10)], four methylenes [ $\delta_{\mathrm{H}} 3.41$ and 3.76 (each $\left.1 \mathrm{H}, \mathrm{m}, \mathrm{H}-1\right), 1.32$ and 1.56 (each $1 \mathrm{H}, \mathrm{m}, \mathrm{H}-2), 1.12$ and 1.29 (each $1 \mathrm{H}, \mathrm{m}, \mathrm{H}-4)$, and $1.93(2 \mathrm{H}, \mathrm{m}, \mathrm{H}-5) ; \delta_{\mathrm{C}} 66.9(\mathrm{C}-1), 36.3$ (C-2), $36.8(\mathrm{C}-4)$, and $24.9(\mathrm{C}-5)$ ], one olefinic methine $\left[\delta_{\mathrm{H}} 1.52(1 \mathrm{H}, \mathrm{m}, \mathrm{H}-3) ; \delta_{\mathrm{C}} 28.9(\mathrm{C}-3)\right]$, and one carbon-carbon double bond $\left[\delta_{\mathrm{H}} 5.09(1 \mathrm{H}, \mathrm{t}\right.$-like, $J=7.2 \mathrm{~Hz}, \mathrm{H}-6) ; \delta_{\mathrm{C}} 124.7$ (C-6) and 130.4 (C-4)]. Based on the 2D NMR data of H-H COSY, HMQC and HMBC experiments, the monoterpene moiety was identified as citronellol [4]. The coupling constant of the anomeric proton of glucose at $\delta 4.11(\mathrm{~d}, 1 \mathrm{H}, J=8.0 \mathrm{~Hz})$ indicated that glucose moiety was in a $\beta$-configuration. The HMBC correlation signal of the anomeric proton of glucose, 1'-H $\left(\delta_{\mathrm{H}} 4.11\right)$ to $\mathrm{C}-1\left(\delta_{\mathrm{C}} 66.9\right)$ showed that glucose was linked to C-1 of the alycone. The downfield shift by about $5 \mathrm{ppm}$ of the signal of C-6' of glucose $\left(\delta_{\mathrm{C}} 67.2\right)$ showed that arabinose was linked to C-6' of the glucose, which was further confirmed by the HMBC correlations of $6^{\prime}-\mathrm{H}\left(\delta_{\mathrm{H}} 3.39\right.$ and 3.85) to $\mathrm{C}-1$ " $\left(\delta_{\mathrm{C}} 108.5\right)$ of arabinose and $1 "-\mathrm{H}\left(\delta_{\mathrm{H}} 4.79\right)$ to $\mathrm{C}-6$ ' $\left(\delta_{\mathrm{C}} 67.2\right)$. The ${ }^{13} \mathrm{C}-\mathrm{NMR}$ signals of compound 1 assignable to the arabinose moiety $\left[\delta_{\mathrm{C}} 82.0(\mathrm{C}-2 "), 83.8(\mathrm{C}-2 ")\right]$ and the coupling constant of the anomeric proton of arabinose at $\delta_{\mathrm{H}} 4.79(\mathrm{~d}, 1 \mathrm{H}, J=1.6 \mathrm{~Hz})$ indicated that arabinose moiety was an $\alpha$ - L -arabinofuranose moiety [5], which was further confirmed by the HMBC correlation of 1 "-H $\left(\delta_{\mathrm{H}} 4.79\right)$ to C-4" $\left(\delta_{\mathrm{C}} 83.8\right)$. The complete assignment of the signals of compound 1 was based on DEPT ${ }^{13} \mathrm{C}-\mathrm{NMR}$ and 2D-NMR H-H COSY, HMQC and HMBC data. For all the ${ }^{1} \mathrm{H}-,{ }^{13} \mathrm{C}-$, and HMBC NMR data of compound 1 see Table 1, and for the structure of compound 1, see Figure 1. Therefore, the structure of compound $\mathbf{1}$ was elucidated as citronellol-1-O- $\alpha$-L-arabinofuranosyl- $(1 \rightarrow 6)-\alpha$-D-glucopyranoside.

Using similar methods as described above, compounds 2-7 were identified as geraniol-1-O- $\alpha-\mathrm{L}-$ arabinofuranosyl-( $1 \rightarrow 6)-\beta$-D-glucopyranoside (2) [5], geraniol-1- $O$ - $\alpha$-L-arabinopyranosyl-(1 $\rightarrow 6)-\beta$-D-

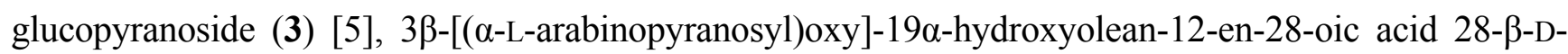

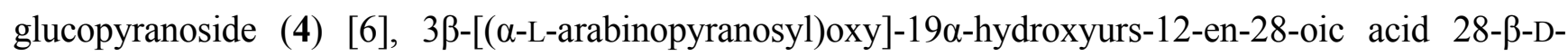

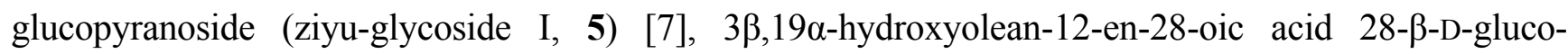

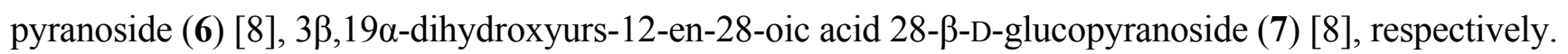


Table 1. ${ }^{1} \mathrm{H}-\mathrm{NMR}(400 \mathrm{MHz}),{ }^{13} \mathrm{C}-\mathrm{NMR}(100 \mathrm{MHz})$, HMQC and HMBC data of compound 1 (DMSO- $\left.\mathrm{d}_{6}, \delta \mathrm{ppm}\right)$.

\begin{tabular}{|c|c|c|c|c|c|c|c|}
\hline No. & $\delta \mathrm{C}$ & $\delta \mathbf{H}$ & HMBC $(H \rightarrow C)$ & No. & $\delta \mathrm{C}$ & $\delta \mathbf{H}$ & $\mathrm{HMBC}(\mathrm{H} \rightarrow \mathrm{C})$ \\
\hline \multicolumn{2}{|c|}{ aglycone } & \multicolumn{5}{|c|}{ glc } & \\
\hline 1 & 66.9 & $\begin{array}{c}3.41,3.76 \\
(\mathrm{~m}, \text { each } 1 \mathrm{H})\end{array}$ & $28.9,102.8$ & $1^{\prime}$ & 102.8 & $4.11(\mathrm{~d}, 1 \mathrm{H}, J=8.0 \mathrm{~Hz})$ & \multirow[t]{3}{*}{$66.9,75.4,76.7$} \\
\hline 2 & 36.3 & $\begin{array}{c}1.32,1.56 \\
(\mathrm{~m}, \text { each } 1 \mathrm{H})\end{array}$ & 19.3, 36.8, & $2^{\prime}$ & 73.4 & $\begin{array}{c}2.93(\mathrm{t}-\mathrm{like}, 1 \mathrm{H} \\
\quad J=8.0 \mathrm{~Hz})\end{array}$ & \\
\hline 3 & 28.9 & $1.52(\mathrm{~m}, 1 \mathrm{H})$ & 66.9 & $3^{\prime}$ & 76.7 & $3.13(\mathrm{t}, 1 \mathrm{H}, J=8.8 \mathrm{~Hz})$ & \\
\hline 4 & 36.8 & $\begin{array}{c}1.12,1.29 \\
(\mathrm{~m}, \text { each } 1 \mathrm{H})\end{array}$ & $\begin{array}{c}19.3,36.3 \\
124.7\end{array}$ & $4^{\prime}$ & 70.4 & $\begin{array}{c}2.98(\mathrm{t}-\mathrm{like}, 1 \mathrm{H} \\
\quad J=8.4 \mathrm{~Hz})\end{array}$ & 73.4 \\
\hline 5 & 24.9 & $1.93(\mathrm{~m}, 2 \mathrm{H})$ & $28.9,130.4$ & $5^{\prime}$ & 75.4 & $\begin{array}{c}3.28(\mathrm{t}-\text { like, } 1 \mathrm{H} \\
\quad J=8.4 \mathrm{~Hz})\end{array}$ & 102.8 \\
\hline 6 & 124.7 & $\begin{array}{c}5.09 \text { (t-like, } 1 \mathrm{H} \\
J=7.2 \mathrm{~Hz})\end{array}$ & $17.5,25.5,36.8$ & $6^{\prime}$ & 67.2 & $\begin{array}{c}3.85(\mathrm{~d}-\text { like, } 1 \mathrm{H} \\
J=10.8 \mathrm{~Hz}), 3.39(\mathrm{dd}, \\
1 \mathrm{H}, J=10.8,8.4 \mathrm{~Hz})\end{array}$ & 108.5 \\
\hline 7 & 130.4 & \multicolumn{6}{|c|}{$\operatorname{ara}(f)$} \\
\hline 8 & 25.5 & $1.64(\mathrm{~s}, 3 \mathrm{H})$ & $17.5,124.7$ & $1 "$ & 108.5 & $4.79(\mathrm{~d}, 1 \mathrm{H}, J=1.6 \mathrm{~Hz})$ & $67.2,77.2,83.8$ \\
\hline 9 & 17.5 & $1.56(\mathrm{~s}, 3 \mathrm{H})$ & $25.5,124.7$ & $2 "$ & 82.0 & $3.79(\mathrm{~m}, 1 \mathrm{H})$ & \\
\hline \multirow[t]{3}{*}{10} & 19.3 & $\begin{array}{l}0.85(\mathrm{~d}, 3 \mathrm{H} \\
J=6.4 \mathrm{~Hz})\end{array}$ & $36.3,36.8$ & $3 "$ & 77.2 & $3.62(\mathrm{~m}, 1 \mathrm{H})$ & \\
\hline & & & & $4 "$ & 83.8 & $3.72(\mathrm{~m}, 1 \mathrm{H})$ & \\
\hline & & & & $5 "$ & 61.4 & $\begin{array}{c}3.55(\mathrm{dd}, 1 \mathrm{H}, J=11.6 \\
2.0 \mathrm{~Hz}), 3.40(\mathrm{~m}, 1 \mathrm{H})\end{array}$ & \\
\hline
\end{tabular}

All assignments based on extensive 1D and 2D NMR experiments (HMQC, HMBC, 1H-1H COSY).

Figure 1. The key HMBC correlations of compound 1.

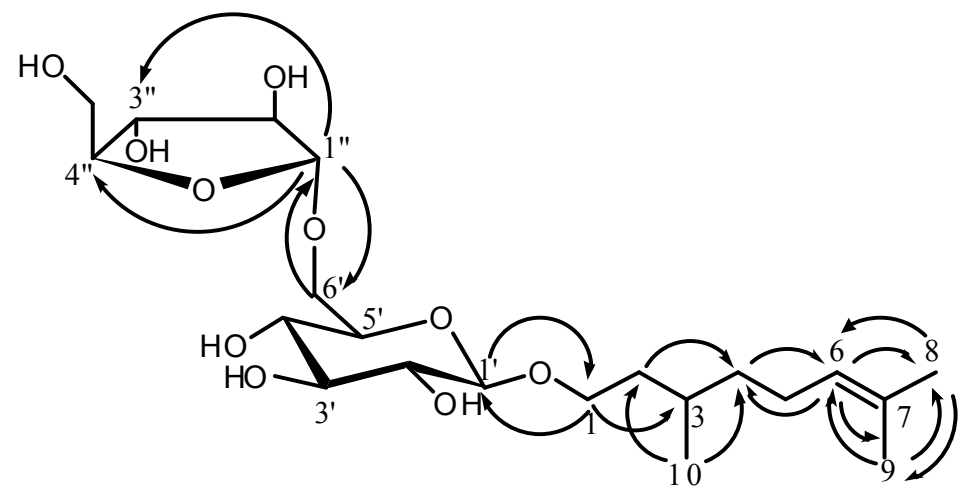

Note: Arrows point from proton to carbon.

Compounds 1-7 were next assayed for hemostatic activity with a Goat Anti-Human $\alpha 2-$ plasmin inhibitor ELISA kit, and the results are shown in Table 2. The data proved that ziyu-glycoside I showed strongest hemostasis activity within 7 kinds of terpene glycosides. It is the first report that ziyu-glycoside I (5) has strong hemostatic activity. 
Table 2. The hemostasis assay data of the separated fractions and the isolated compounds.

\begin{tabular}{|c|c|c|c|c|c|c|c|c|c|c|}
\hline Fractions & $\mathrm{H}_{2} \mathrm{O}$ & $\begin{array}{c}30 \% \\
\text { EtOH }\end{array}$ & $\begin{array}{c}70 \% \\
\text { EtOH } \\
\end{array}$ & $\begin{array}{c}95 \% \\
\text { EtOH } \\
\end{array}$ & I & II & III & IV & $\begin{array}{c}\text { Control } \\
\text { blank } \\
\end{array}$ & $\begin{array}{c}\text { Standard } \\
(10 \mathrm{~g} / \mathrm{L})\end{array}$ \\
\hline $\begin{array}{c}\text { OD value } \\
(\mathrm{n}=3)\end{array}$ & $\begin{array}{c}0.056 \pm \\
0.011 \\
\end{array}$ & $\begin{array}{c}0.053 \pm \\
0.002 \\
\end{array}$ & $\begin{array}{c}0.131 \pm \\
0.014 \\
\end{array}$ & $\begin{array}{c}0.051 \pm \\
0.006 \\
\end{array}$ & $\begin{array}{c}0.051 \pm \\
0.005 \\
\end{array}$ & $\begin{array}{c}0.051 \pm \\
0.006 \\
\end{array}$ & $\begin{array}{c}0.490 \pm \\
0.017 \\
\end{array}$ & $\begin{array}{c}0.061 \pm \\
0.004 \\
\end{array}$ & $\begin{array}{c}0.028 \pm \\
0.004\end{array}$ & $\begin{array}{c}0.828 \pm \\
0.031 \\
\end{array}$ \\
\hline $\begin{array}{c}\text { Percent } \\
\text { inhibition }\end{array}$ & 3.5 & 3.1 & 12.8 & 2.9 & 2.9 & 2.9 & 57.8 & 4.1 & & \\
\hline Compounds & 1 & 2 & 3 & 4 & 5 & 6 & 7 & & $\begin{array}{c}\text { Control } \\
\text { blank }\end{array}$ & $\begin{array}{c}\text { Standard } \\
(10 \mathrm{~g} / \mathrm{L})\end{array}$ \\
\hline $\begin{array}{l}\text { OD value } \\
(\mathrm{n}=3)\end{array}$ & $\begin{array}{c}0.138 \pm \\
0.016 \\
\end{array}$ & $\begin{array}{c}0.111 \pm \\
0.009 \\
\end{array}$ & $\begin{array}{c}0.122 \pm \\
0.003 \\
\end{array}$ & $\begin{array}{c}0.260 \pm \\
0.013 \\
\end{array}$ & $\begin{array}{c}0.741 \pm \\
0.012 \\
\end{array}$ & $\begin{array}{c}0.227 \pm \\
0.010 \\
\end{array}$ & $\begin{array}{c}0.214 \pm \\
0.015 \\
\end{array}$ & & $\begin{array}{c}0.060 \pm \\
0.004 \\
\end{array}$ & $\begin{array}{c}0.828 \pm \\
0.031 \\
\end{array}$ \\
\hline $\begin{array}{c}\text { Percent } \\
\text { inhibition }\end{array}$ & 10.2 & 6.6 & 8.1 & 26.0 & 88.7 & 21.7 & 20.1 & & & \\
\hline
\end{tabular}

Note: Percent inhibition $=\left[\left(\mathrm{OD}_{\text {sample }}-\mathrm{OD}_{\text {blank }}\right) \div\left(\mathrm{OD}_{\text {standard }}-\mathrm{OD}_{\text {blank }}\right)\right] \times 100$; the $\mathrm{OD}$ value is directly proportional the concentration of $\alpha 2$-plasmin inhibitor present in the samples.

\section{Experimental}

\subsection{General}

IR spectra were recorded on a FT-IR 5DX Nicolet/Nicolet Magna IR-560 spectrometer (Thermo Scientific, Osaka, Japan). ${ }^{1} \mathrm{H}$ - and ${ }^{13} \mathrm{C}-\mathrm{NMR}$ spectra were recorded on a Bruker AV-400 spectrometer (Zürich, Switzerland). HR-ESI-MS were recorded on a Bruker microOTOF-Q II mass spectrometer. Prep. HPLC was performed on a Shimadzu LC-10A equipped with a SPD-10A detector and Gemini $5 \mu \mathrm{m} \mathrm{C} 18$ 110A column $(250 \mathrm{~mm} \times 10.00 \mathrm{~mm}, 5 \mu \mathrm{m}$, flow rate: $3.0 \mathrm{~mL} / \mathrm{min})$. The bioactivities were measured on a DG5033A Enzyme immunoassay spectrophotometer (Nanjing, China), using Goat Anti-Human a2-plasmin inhibitor ELISA kit [96/48-wells microtiter plates, plastic cover, standard (40 g/L), blank control, standard diluent, biotinylated anti- $\alpha 2$-plasmin inhibitor, streptavidin-HRP, washing buffer, substrate A, substrate B, stopping solution, sample diluent)] (R\&D Systems, Minneapolis, MN, USA). The roots of S. officinalis L. were collected in Tong-Hua County in Jilin Province, China. They were identified by Prof. Jing-min Zhang of School of Pharmaceutical Sciences, Jilin University.

\subsection{Extraction and Isolation}

The air-dried the roots of $S$. officinalis $(4.0 \mathrm{~kg}$ ) were extracted with hot $70 \% \mathrm{EtOH}$ (ca. $20 \mathrm{~L}, 24 \mathrm{~h}$, $\left.45^{\circ} \mathrm{C}\right)$. The EtOH extract was concentrated under reduced pressure, and the viscous concentrate $(420 \mathrm{~g})$ was passed through a D101 polyporous resin column eluting successively with $\mathrm{H}_{2} \mathrm{O}, 30 \%$ EtOH, 70\% $\mathrm{EtOH}$, and 95\% EtOH, and by vacuum distillation recovery, four fractions (I-IV) were obtained. The bioassay experiments suggested that the $70 \% \mathrm{EtOH}$ portion had hemostatic activity. Therefore the $70 \%$ EtOH fraction was subjected to silica gel column chromatography eluted with a stepwise gradient mixture of $\mathrm{CHCl}_{3}-\mathrm{MeOH}(9: 1 ; 6: 1 ; 3: 1)$, and finally with $\mathrm{MeOH}$ alone, and four fractionons (I-IV) were obtained. Fraction III having hemostasis activity was further subjected to a silica gel column eluted with $\mathrm{CHCl}_{3}-\mathrm{MeOH}-\mathrm{EtOAc}-\mathrm{H}_{2} \mathrm{O}$ (6.5:5:4:1.7), and three fractions (A, B, C) were obtained. 
Fraction B was applied to a ODS-A $(50 \mu \mathrm{m}, 12 \mathrm{~nm}, \mathrm{YMC}$, Kyoto, Japan) column eluted with a stepwise gradient mixture of $\mathrm{MeOH}-\mathrm{H}_{2} \mathrm{O}(2: 3 ; 3: 2 ; 4: 1)$, and thus compound 5 (230 $\mathrm{mg}$ ) and Fraction D were obtained. Fractions A, C, and D were separated by preparative HPLC using $\mathrm{MeOH}-\mathrm{H}_{2} \mathrm{O}$ (60:40, 70:30, 65:35, respectively), and compound $\mathbf{1}(22 \mathrm{mg}), \mathbf{2}(25 \mathrm{mg})$, and $\mathbf{3}(27 \mathrm{mg})$ were isolated from fraction A, compound $6(30 \mathrm{mg})$ and $7(33 \mathrm{mg})$ from fraction $C$, and compound 4 (30 $\mathrm{mg})$ from Fraction B.

Compound 1: Colorless amorphous powder, $[\alpha]_{D}^{24}:-21.0^{\circ}$ (c 0.6, MeOH). HRESIMS, $m / z: 473.2369$ $\left([\mathrm{M}+\mathrm{Na}]^{+}\right.$; calcd for $\left.\mathrm{C}_{21} \mathrm{H}_{38} \mathrm{O}_{10} \mathrm{Na}, 473.2363\right)$. IR (KBr) $v_{\max }: 3450 \mathrm{~cm}^{-1} .{ }^{1} \mathrm{H}$ and ${ }^{13} \mathrm{C}-\mathrm{NMR}$ : See Table 1 .

Compound 2: Colorless amorphous powder. HRESIMS, $m / z: 471.2209\left([\mathrm{M}+\mathrm{Na}]^{+}\right.$; calcd for $\left.\mathrm{C}_{21} \mathrm{H}_{36} \mathrm{O}_{10} \mathrm{Na}, 471.2206\right)$. IR (KBr) $v_{\max }: 3440 \mathrm{~cm}^{-1} .{ }^{1} \mathrm{H}-\mathrm{NMR}$ (DMSO-d 6 ) $\delta: 1.57$ (s, 3H, H-9), 1.61 (s, $3 \mathrm{H}, \mathrm{H}-10), 1.64$ (s, 3H, H-8), 1.99 (2H, t, $J=6.8 \mathrm{~Hz}, \mathrm{H}-4), 2.05$ (2H, m, H-5), 4.08 (1H, dd, $J=11.9$, $7.6 \mathrm{~Hz}, \mathrm{H}-1 \mathrm{a}), 4.19(1 \mathrm{H}, \mathrm{dd}, J=11.9,6.1 \mathrm{~Hz}, \mathrm{H}-1 \mathrm{~b}), 5.07$ (1H, t-like, $J=6.8 \mathrm{~Hz}, \mathrm{H}-6), 5.26$ (1H, m, $\mathrm{H}-2) ; 4.11(1 \mathrm{H}, \mathrm{d}, J=7.8 \mathrm{~Hz}$, glc-H1), $2.95(1 \mathrm{H}, \mathrm{t}-$ like, $J=8.0 \mathrm{~Hz}$, glc-H2), $3.12(1 \mathrm{H}, \mathrm{t}-\mathrm{like}, J=8.8 \mathrm{~Hz}$, glc-H3), 2.98 (1H, t-like, $J=8.8 \mathrm{~Hz}$, glc-H4), 3.25 (1H, t-like, $J=8.4 \mathrm{~Hz}$, glc-H5), 3.86 (1H, d-like, $J=9.9 \mathrm{~Hz}$, glc-H6a), 3.39 (1H, m, glc-H6b); $4.79(1 \mathrm{H}, \mathrm{d}, J=1.6 \mathrm{~Hz}$, ara-H1), $3.79(1 \mathrm{H}, \mathrm{m}$, ara-H2), $3.62(1 \mathrm{H}, \mathrm{m}$, ara-H3), $3.72(1 \mathrm{H}, \mathrm{m}$, ara-H4), $3.52(1 \mathrm{H}, \mathrm{d}-\mathrm{like}, J=13.4 \mathrm{~Hz}$, ara-H5a),3.40 (m, $1 \mathrm{H}$, ara-H5b). ${ }^{13} \mathrm{C}-\mathrm{NMR}$ (DMSO-d 6 ) $\delta:$ 16.1(C-10), 17.6 (C-9), 25.6 (C-8), 25.9 (C-5), 39.4(C-4), 64.2 (C-1), 120.6 (C-2), 123.9 (C-6), 130.4 (C-7), 139.4 (C-3); 101.3 (glc-C1), 73.3 (glc-C2), 76.7 (glc-C3), 70.5 (glc-C4), 75.5 (glc-C5), 67.2 (glc-C6); 108.5 (ara(f)-C1), 82.1 (ara(f)-C2), 77.3 (ara(f)-C3), 83.8 $(\operatorname{ara}(\mathrm{f})-\mathrm{C} 4), 61.4(\operatorname{ara}(\mathrm{f})-\mathrm{C} 4)$.

Compound 3: Colorless amorphous powder. HRESIMS, $m / z: 471.2208\left([\mathrm{M}+\mathrm{Na}]^{+}\right.$; calcd for $\mathrm{C}_{21} \mathrm{H}_{36} \mathrm{O}_{10} \mathrm{Na}, 471.2206$ ). IR (KBr) $v_{\max }: 3445 \mathrm{~cm}^{-1} .{ }^{1} \mathrm{H}-\mathrm{NMR}$ (DMSO-d 6 ) $\delta: 1.57$ (s, 3H, H-9), 1.61 (s, 3H, H-10), 1.64 (s, 3H, H-8), 1.99 (2H, t, $J=6.8 \mathrm{~Hz}, \mathrm{H}-4), 2.05$ (2H, t, $J=6.8 \mathrm{~Hz}, \mathrm{H}-5), 4.06$ (1H, dd, $J=12.7,7.3 \mathrm{~Hz}, \mathrm{H}-1 \mathrm{a}), 4.22(1 \mathrm{H}, \mathrm{dd}, J=12.7,6.0 \mathrm{~Hz}, \mathrm{H}-1 \mathrm{~b}), 5.08(1 \mathrm{H}, \mathrm{t}-\mathrm{like}, J=6.5 \mathrm{~Hz}, \mathrm{H}-6), 5.28$ $(1 \mathrm{H}, J=6.5 \mathrm{~Hz}, \mathrm{H}-2) ; 4.12\left(1 \mathrm{H}, \mathrm{d}, J=7.8 \mathrm{~Hz}\right.$, glc-H1), $4.20\left(1 \mathrm{H}, \mathrm{d}, J=6.0 \mathrm{~Hz}\right.$, ara-H1). ${ }^{13} \mathrm{C}-\mathrm{NMR}$ $\left(\mathrm{DMSO}_{6}\right.$ ) $\delta$ : 16.1 (C-10), 17.6 (C-9), 25.5 (C-8), 25.8 (C-5), 39.3 (C-4), 64.4 (C-1), 120.7 (C-2), 123.9 (C-6), 130.9 (C-7), 139.1 (C-3); 101.5 (glc-C1), 73.3 (glc-C2), 76.6 (glc-C3), 70.5 (glc-C4), 75.6 (glc-C5), 67.2 (glc-C6); 103.4 (ara(p)-C1), 70.1 (ara(p)-C2), 72.5 (ara(p)-C3), 68.5(ara(p)-C4), 64.7 $(\operatorname{ara}(\mathrm{p})-\mathrm{C} 4)$.

Compound 4: Colorless amorphous powder. HRESIMS, $m / z$ : $789.4404\left([\mathrm{M}+\mathrm{Na}]^{+}\right.$; calcd for $\left.\mathrm{C}_{41} \mathrm{H}_{66} \mathrm{O}_{13} \mathrm{Na}, 789.4401\right)$. IR (KBr) $v_{\max }: 3440,1720 \mathrm{~cm}^{-1} .{ }^{1} \mathrm{H}-\mathrm{NMR}$ (DMSO-d $\left.\mathrm{d}_{6}\right) \delta: 5.24(1 \mathrm{H}, \mathrm{d}$, $J=7.8 \mathrm{~Hz}$, glc-H1), 5.23(1H, br.s, H-12), 4.45 (1H, d, $J=7.0 \mathrm{~Hz}$, ara-H1), 3.11 (1H, br.s, H-19), 3.01 $(1 \mathrm{H}, \mathrm{dd}, J=11.5,3.9 \mathrm{~Hz}, \mathrm{H}-3), 2.91(1 \mathrm{H}$, br.s, H-18), 1.23 (3H, s, Me-27), 0.97 (3H, s, Me-23), 0.87 $(3 \mathrm{H} \times 2, \mathrm{~s}, \mathrm{Me}-26$ and Me-29), $0.85(3 \mathrm{H}, \mathrm{s}, \mathrm{Me}-30), 0.76(3 \mathrm{H}, \mathrm{s}, \mathrm{Me}-24), 0.65(3 \mathrm{H}, \mathrm{s}, \mathrm{Me}-25)$. ${ }^{13} \mathrm{C}-\mathrm{NMR}\left(\mathrm{DMSO}_{6}\right.$ ) $\delta:$ 38.0(C-1), 25.6 (C-2), 87.7 (C-3), 38.8 (C-4), 55.1 (C-5), 17.9 (C-6), 32.3 (C-7), 40.2 (C-8), 47.3 (C-9), 36.4 (C-10); 23.2 (C-11), 122.3 (C-12), 143.2 (C-13), 41.1 (C-14), 28.3 (C-15), 27.0 (C-16); 45.2 (C-17), 43.2 (C-18), 80.0 (C-19), 34.8 (C-20), 27.8 (C-21), 31.8 (C-22), 27.6 (C-23), 16.4 (C-24), 15.1 (C-25), 16.6 (C-26); 24.5 (C-27), 175.8 (C-28), 28.0 (C-29), 24.0 (C-30); 
94.1 (glc-C1), 72.4 (glc-C2), 76.7 (glc-C3), 69.5 (glc-C4), 77.7 (glc-C5), 60.6 (glc-C6); 105.8 (ara(p)C1), 71.0 (ara(p)-C2), 72.7 (ara(p)-C3), 67.6 (ara-C4), 65.1 (ara-C4).

Compound 5: Colorless amorphous powder. HRESIMS, $m / z$ : $789.4403\left([\mathrm{M}+\mathrm{Na}]^{+}\right.$; calcd for $\left.\mathrm{C}_{41} \mathrm{H}_{66} \mathrm{O}_{13} \mathrm{Na}, 789.4401\right)$. IR (KBr) $v_{\max }: 3475,1740 \mathrm{~cm}^{-1} .{ }^{1} \mathrm{H}-\mathrm{NMR}$ (DMSO-d $\left.\mathrm{d}_{6}\right) \delta: 5.16(1 \mathrm{H}, \mathrm{d}$, $J=7.8 \mathrm{~Hz}$, glc-H1), 5.17(1H, br.s, H-12), $4.12(1 \mathrm{H}, \mathrm{d}, J=6.0 \mathrm{~Hz}$, ara-H1), 3.11 (1H, br.s, H-19), 3.01 (1H, dd, $J=11.1,3.9 \mathrm{~Hz}, \mathrm{H}-3), 2.36(1 \mathrm{H}$, br.s, H-18), 1.23 (3H, s, Me-27), 1.09 (3H, s, Me-29), 0.97 (3H, s, Me-23), 0.88 (3H, s, Me-26), 0.84(3H, d, $J=6.6 \mathrm{~Hz}, \mathrm{Me}-30), 0.76$ (3H, s, Me-25), 0.67 (3H, s, Me-24). ${ }^{13} \mathrm{C}-\mathrm{NMR}$ (DMSO-d ${ }_{6}$ ) : $38.2(\mathrm{C}-1), 25.7$ (C-2), 87.8 (C-3), 38.7 (C-4), 55.0 (C-5), 17.9 (C-6), 32.5 (C-7), 39.4 (C-8), 46.7 (C-9), 36.2 (C-10); 23.2 (C-11), 127.0 (C-12), 138.2 (C-13), 41.2 (C-14), 28.1 (C-15), 25.1 (C-16); 47.3 (C-17), 53.2 (C-18), 71.6 (C-19), 41.0 (C-20), 25.8 (C-21), 36.6 (C-22), 27.6 (C-23), 16.4 (C-24), 15.2 (C-25), 16.4 (C-26); 23.8 (C-27), 175.5 (C-28), 26.4 (C-29), 16.2 (C-30); 94.1 (glc-C1), 72.2 (glc-C2), 76.7 (glc-C3), 69.5 (glc-C4), 77.6 (glc-C5), 60.6 (glc-C6); 105.8 (ara(p)-C1), $71.0(\operatorname{ara}(\mathrm{p})-\mathrm{C} 2), 72.7$ (ara(p)-C3), 67.6 (ara-C4), 65.1 (ara-C4).

Compound 6: Colorless amorphous powder. HRESIMS, $m / z$ : $657.39783\left([\mathrm{M}+\mathrm{Na}]^{+}\right.$; calcd for $\left.\mathrm{C}_{36} \mathrm{H}_{58} \mathrm{O}_{9} \mathrm{Na}, 657.39785\right)$. IR (KBr) $v_{\max }: 3440,1720 \mathrm{~cm}^{-1} .{ }^{1} \mathrm{H}-\mathrm{NMR}$ (DMSO-d $\left.{ }_{6}\right) \delta: 5.23(1 \mathrm{H}, \mathrm{d}$, $J=7.6 \mathrm{~Hz}$, glc-H1), 5.23(1H, br.s, H-12), $3.11(1 \mathrm{H}$, br.s, H-19), $3.00(1 \mathrm{H}, \mathrm{dd}, J=11.2,3.9 \mathrm{~Hz}$, H-3), 2.91(1H, br.s, H-18), 1.23 (3H, s, Me-27), 0.89 (3H, s, Me-23), 0.87 (3H, s, Me-29), 0.84 $\left(3 \mathrm{H} \times 2, \mathrm{~s}, \mathrm{Me}-25\right.$ and Me-30), $0.68(3 \mathrm{H}, \mathrm{s}, \mathrm{Me}-24), 0.64(3 \mathrm{H}, \mathrm{s}, \mathrm{Me}-25) .{ }^{13} \mathrm{C}-\mathrm{NMR}\left(\mathrm{DMSO}-\mathrm{d}_{6}\right) \delta: 38.0$ (C-1), 26.9 (C-2), 77.7 (C-3), 38.4 (C-4), 54.9 (C-5), 18.1 (C-6), 32.3 (C-7), 40.2 (C-8), 47.3 (C-9), 36.7 (C-10); 23.2 (C-11), 122.3 (C-12), 143.2 (C-13), 41.1 (C-14), 28.3 (C-15), 27.0 (C-16); 45.2 (C-17), 43.1 (C-18), 80.0 (C-19), 34.8 (C-20), 27.8 (C-21), 31.8 (C-22), 28.3 (C-23), 15.9 (C-24), 15.0 (C-25), 16.6 (C-26); 24.1 (C-27), 175.8 (C-28), 28.0 (C-29), 24.5 (C-30); 94.1 (glc-C1), 72.4 (glc-C2), 76.7 (glc-C3), 69.5 (glc-C4), 76.9 (glc-C5), 60.6 (glc-C6).

Compound 7: Colorless amorphous powder. HRESIMS, $m / z: 657.39784\left([\mathrm{M}+\mathrm{Na}]^{+}\right.$; calcd for $\mathrm{C}_{36} \mathrm{H}_{58} \mathrm{O}_{9} \mathrm{Na}$, 657.39785). IR (KBr) $v_{\max }: 3475,1740 \mathrm{~cm}^{-1} .{ }^{1} \mathrm{H}-\mathrm{NMR}$ (DMSO-d $\left.{ }_{6}\right) \delta: 5.16(1 \mathrm{H}, \mathrm{d}$, $J=8.0 \mathrm{~Hz}$, glc-H1), 5.17(1H, br.s, H-12), $4.12(1 \mathrm{H}, \mathrm{d}, J=6.0 \mathrm{~Hz}$, ara-H1), 3.11 (1H, br.s, H-19), 3.00 $(1 \mathrm{H}, \mathrm{dd}, J=11.0,3.9 \mathrm{~Hz}, \mathrm{H}-3), 2.37(1 \mathrm{H}$, br.s, H-18), 1.27 (3H, s, Me-27), 1.08 (3H, s, Me-29), 0.89 (3H, s, Me-23), 0.85 (3H, s, Me-26), $0.84(3 \mathrm{H}, \mathrm{d}, J=6.6 \mathrm{~Hz}, \mathrm{Me}-30),(3 \mathrm{H}, \mathrm{s}),, 0.67(3 \mathrm{H} \times 2, \mathrm{~s}, \mathrm{Me}-25$ and Me-24). ${ }^{13} \mathrm{C}-\mathrm{NMR}\left(\mathrm{DMSO}_{6}\right)$ ) 38.2 (C-1), 27.0 (C-2), 77.6 (C-3), $38.4(\mathrm{C}-4), 54.8$ (C-5), 18.1 (C-6), 32.6 (C-7), 39.2 (C-8), 46.7 (C-9), 36.5 (C-10); 23.2 (C-11), 127.0 (C-12), 138.1 (C-13), 41.2 (C-14), 28.1 (C-15), 25.1 (C-16); 47.3 (C-17), 53.2 (C-18), 71.6 (C-19), 41.1 (C-20), 25.4 (C-21), 36.6 (C-22), 28.2 (C-23), 16.4 (C-24), 15.1 (C-25), 16.2 (C-26); 23.8 (C-27), 175.5 (C-28), 26.4 (C-29), 16.0 (C-30); 94.0 (glc-C1), 72.2 (glc-C2), 76.7 (glc-C3), 69.5 (glc-C4), 76.9 (glc-C5), 60.6 (glc-C6).

\subsection{Acid Hydrolysis of $\mathbf{1}-\mathbf{7}$}

Solutions of 1-7 (each $1.0 \mathrm{mg}$ ) in $0.5 \mathrm{M} \mathrm{H}_{2} \mathrm{SO}_{4}(2.0 \mathrm{~mL}$ ) were heated under reflux for $3 \mathrm{~h}$. After cooling, each reaction mixture was diluted with $\mathrm{H}_{2} \mathrm{O}$, neutralized with $\mathrm{BaCO}_{3}$, then filtered. The solution was partitioned with EtOAc to give two layers. The aqueous layer was evaporated and then subjected to TLC analysis with authentic sugar samples using $n$ - $\mathrm{BuOH}-\mathrm{MeOH}-\mathrm{CHCl}_{3}-\mathrm{HOAc}$ 
(12.5:4.5:9:1.5:1, detection with aniline-phthalic acid). Compounds $1-5$ afforded D-glucose $\left(\mathrm{R}_{\mathrm{f}}=0.30\right)$ and L-arabinose $\left(\mathrm{R}_{\mathrm{f}}=0.36\right)$, and $\mathbf{6}-\mathbf{7}$ gave $\mathrm{D}$-glucose $\left(\mathrm{R}_{\mathrm{f}}=0.30\right)$.

\subsection{Bioactivity Assay}

The hemostasis assay was carried out by using a Goat Anti-Human $\alpha 2$-plasmin inhibitor kit which is a solid phase sandwich enzyme-linked quantitative immunoabsorbent assay (ELISA) with a purified antibody specific for $\alpha 2$-plasmin inhibitors. The above separated fractions and compounds were dissolved in dimethylsulfoxide (DMSO) $(0.188 \mathrm{mg} / \mu \mathrm{L}$ for the fractions; $0.094 \mathrm{mg} / \mu \mathrm{L}$ for compounds), and were diluted with sample diluent (1:1). After an aliquot $(50 \mu \mathrm{L})$ of the above samples or standards was added to each microplate well, a portion of biotinylated anti- $\alpha 2$-plasmin inhibitor $(50 \mu \mathrm{L})$ was immediately added to each well, and the microplate was incubated for $1 \mathrm{~h}$ at $37^{\circ} \mathrm{C}$. After a wash with washing buffer, streptavidin-HRP was added, and the microplate was incubated for $30 \mathrm{~min}$ at $37^{\circ} \mathrm{C}$. Again, after a wash with washing buffer, substrate A and substrate B (50 $\mu \mathrm{L}$ each) were added to each well, and the microplate was incubated for $10 \mathrm{~min}$ at $37{ }^{\circ} \mathrm{C}$. The enzyme-substrate reaction was stopped by quickly pipetting stopping solution $(50 \mu \mathrm{L})$ into each well. The optical density absorbance (OD value) of each well was measured at $450 \mathrm{~nm}$ wavelength on a DG5033A enzyme immunoassay spectrophotometer. All the separated fractions and isolated compounds were tested for their hemostasis activities, and the results are summarized in Table 2.

The correlation of calibration curve test data is shown in Table 3. By using OD value as $\mathrm{Y}$-axis(Y) and standards cocentration as $\mathrm{X}$-axis $(\mathrm{X})$, the linear regression equation, $\mathrm{y}=0.0687 \mathrm{x}+0.0317$, $\mathrm{r}=0.9997$, was obtained. The results showed that the linear relation between OD value and concentration of standards were good and linearity domain of the measure is $0 \sim 40 \mathrm{~g} / \mathrm{L}$. Therefore the OD value is directly proportional the concentration of $\alpha 2$-plasmin inhibitor present in the samples.

Table 3. The correlation of calibration curve test data.

\begin{tabular}{|c|c|c|c|c|c|c|c|}
\hline $\begin{array}{c}\text { Standards } \\
\text { Concentration (g/L) }\end{array}$ & $\mathbf{4 0}$ & $\mathbf{2 0}$ & $\mathbf{1 0}$ & $\mathbf{5 . 0}$ & $\mathbf{2 . 5}$ & $\mathbf{1 . 2 5}$ & $\begin{array}{c}\text { Control } \\
\text { blank }\end{array}$ \\
\hline OD & 2.9404 & 1.5171 & 0.828 & 0.4905 & 0.2955 & 0.0895 & 0.0214 \\
\hline
\end{tabular}

Note: $\mathrm{Y}=0.0687 \mathrm{x}+0.0317, \mathrm{r}=0.9997$, linearity domain: $0 \sim 40 \mathrm{~g} / \mathrm{L}$; the $\mathrm{OD}$ value is directly proportional the concentration of $\alpha 2$-plasmin inhibitor present in the samples.

\section{Conclusions}

Compound $\mathbf{1}$ is a new monoterpene glycoside and compounds 2, $\mathbf{3}$ and $\mathbf{5}$ were isolated from the Sanguisorba genus for the first time. Compounds 1-7 were assayed for their hemostatic activities with a Goat Anti-Human a2-plasmin inhibitor ELISA kit, and the hemostatic constituent of S. officinalis wasidentified as ziyu-glycoside I (5). This is the first report that ziyu-glycoside I has strong hemostatic activity. 


\section{Acknowledgments}

This work was supported by National Science and Technology Major Project of China (2009ZX09502-011). The authors gratefully thank Jing-Min Zhang (School of Pharmaceutical Sciences, Jilin University) for the identification of plants.

\section{References and Notes}

1. The Editorial Board of Zhong Hua Ben Cao of State Administration of Traditional Chinese Medicine of the People's Republic of China. Zhong Hua Ben Cao 4, 1st ed.; Scientific and Technical Publishers: Shanghai, China, 1999; p. 281.

2. Yu, B.B.; Zhong, F.X.; Dong, X. Progress on chemical ingredient of Sanguisorba officinalis L.. Chin. J. Inf. TCM 2009, 16 (Suppl.), 103-105.

3. Xia, H.M.; Sun, L.L.; Sun, J.Y.; Zhong, Y. Progress on chemical ingredient and pharmacological activity of Sanguisorba officinalis L. Food Drug 2009, 11, 67-69.

4. Nakamura, S.; Li, X.Z.; Matsuda, H.; Yoshikawa, M. Bioactive constituents from Chinese natural medicines. XXVIII. Chemical structures of acyclic alcohol glycosides from the roots of Rhodiola crenulata. Chem. Pharm. Bull. 2008, 56, 536-540.

5. Ji, C.J.; Tan, N.H.; Fu, J.; Zhang, Y.M.; He, M. Monoterpene disaccharide glycosides from Rodgersia pinnata. Acta Bot. Yunnanica (Yunnan Zhiwu Yanjiu) 2004, 26, 465-470.

6. Mimaki, Y.; Fukushima, M.; Yokosuka, A.; Sashida, Y.; Furuya, S.; Sakagami, H. Triterpene glycosides from the roots of Sanguisorba officinalis. Phytochemistry 2001, 57, 773-779.

7. Luo, Y.; Wang, H.; Yuan, Z. Triterpenoid saponins of Sanguisorba officinalis and their antiinflammatory activity. Chin. J. Med. Chem. 2008, 18, 138-141.

8. Wu, Z.J.; Ouyang, M.A.; Wang, C.Z.; Zhang, Z.K.; Shen, J.G. Anti-tobacco mosaic virus (TMV) triterpenoid saponins from the leaves of Ilex oblonga. J. Agric. Food Chem. 2007, 55, 1712-1717.

Sample Availability: Samples of the compounds 5 are available from the authors.

(C) 2012 by the authors; licensee MDPI, Basel, Switzerland. This article is an open access article distributed under the terms and conditions of the Creative Commons Attribution license (http://creativecommons.org/licenses/by/3.0/). 